\title{
Molecular characterization of Cryptosporidium and Giardia occurring in natural water bodies in Poland
}

\author{
Malgorzata Adamska
}

Received: 30 September 2014 / Accepted: 14 November 2014 / Published online: 5 December 2014

(C) The Author(s) 2014. This article is published with open access at Springerlink.com

\begin{abstract}
Cryptosporidium and Giardia protozoa are zoonotic parasites that cause human gastroenteritis and can be transmitted to human through the fecal-oral route and water or food. Several species belong to these genera and their resistant forms occur in water, but only some of them are infectious to human. Health risk depends on the occurrence of infectious Cryptosporidium and Giardia species and genotypes in water, and only molecular techniques allow detecting them, as well as enable to identify the contamination source. In this work, genotyping and phylogenetic analysis have been performed on the basis of $18 S$ rDNA and $\beta$-giardin genes sequences of Cryptosporidium and Giardia, respectively, in order to provide the molecular characterization of these parasites detected earlier in five natural water bodies in Poland and to track possible sources of their (oo)cysts in water. Genotyping revealed a high similarity (over 99 up to $100 \%$ ) of analyzed sequences to cattle genotype of C. parvum isolated from cattle and human and to G. intestinalis assemblage B isolated from human. The sequences obtained by others originated from patients with clinical symptoms of cryptosporidiosis or giardiasis and/or with the infection confirmed by different methods. The contamination of three examined lakes is probably human-originated, while the sources of contamination of two remaining lakes are wild and domestic animals. Obtained phylogenetic trees support suggestions of other authors that the bovine genotype of $C$. parvum should be a separate species, as well as A and B assemblages of $G$. intestinalis.
\end{abstract}

M. Adamska $(\square)$

Department of Genetics, Szczecin University, Felczaka 3c,

71-412 Szczecin, Poland

e-mail: adamska.us@wp.pl

M. Adamska

e-mail: genetyka@univ.szczecin.pl
Keywords Cryptosporidium parvum - Giardia intestinalis . Natural water bodies · Genotyping · Phylogenetic analysis . Contamination source

\section{Introduction}

Cryptosporidium is a genus including at least 25 species and 60 genotypes, however, not all of them cause the same level of risk to human (2010; Rossle and Latif 2013; Šlapeta 2013). Cryptosporidium hominis and Cryptosporidium parvum are responsible for over $90 \%$ of cryptosporidiosis cases in human (Rossle and Latif 2013). Moreover, other species have been detected in immunocompetent humans (Rossle and Latif 2013), so there is a threat of zoonotic transmission in the environment. The genotyping techniques can differentiate Cryptosporidium species to detect those infecting human, as well as to track sources of contamination, because most Cryptosporidium species and genotypes are host specific (Xiao and Feng 2008). The genus Giardia can be differentiated into at least six species based on their DNA polymorphism (Ryan and Cacciò 2013) and only Giardia intestinalis infects humans. This one is a complex species that contains eight distinct genotypes (assemblages) and only two of them (A and B) are associated with human infection and have also been found in different species of other mammals (Dado et al. 2012; Ryan and Cacciò 2013). The genotypes cannot be distinguished on the basis of host origin or parasite morphology (Xiao and Fayer 2008), so genotyping is the most useful method for identification of the assemblages infective for human.

The presence of Cryptosporidium spp. oocysts and Giardia spp. cysts in water is an increasing problem throughout the world, and these protozoa are causes of widespread gastrointestinal diseases and morbidity in human and animals (Hajdušek et al. 2004; Xiao and Fayer 2008; Ruecker et al. 
2012; Rossle and Latif 2013; Ryan and Cacciò 2013). The precise identification of a parasite at the species and/or genotype level is of a great importance for various aspects of human and veterinary parasitology, including taxonomy, diagnosis, and treatment (Ruecker et al. 2012), and genotyping is necessary to evaluate the risk of infection for both human and animals. For these reasons, genotyping and phylogenetic analysis have been performed in order to provide the molecular characterization of Cryptosporidium and Giardia detected earlier (Adamska et al. 2014) in natural water bodies in Poland.

\section{Materials and methods}

The sequences of Cryptosporidium 18S rDNA gene and Giardia $\beta$-giardin gene analyzed in this study were obtained earlier from the water samples collected from 36 natural water bodies in north-western Poland (Adamska et al. 2014). Afterwards, they were aligned with published homology sequences with Mega 5.10 software by using ClustalW (Tamura et al. 2011), and they have been deposited in the GenBank database under accession numbers KC748017-23. The sequences were also used for phylogenetic analysis with sequences obtained by others (AB441688, AF093490, AF112572, AF115377, AJ493074, AJ493079, AJ493084, AJ493531, AJ849462, AY120901, AY268583, AY458613, DQ182559, DQ523510, DQ898159, DQ898160, FJ262725, GQ227705, JQ250803, KC608024 for Cryptosporidium and FJ009207, DQ090530, JQ684217, DQ116622, DQ116615, DQ116606, EU189373, EU189369, EU216429, AY072724, JQ684209, JQ247032, AY655702, HQ538712, DQ648780, AY258618 for Giardia). Before the phylogenetic analysis, the ends of the alignments were trimmed in order to form blunt ends on all the sequences in the alignment. The final alignments covered 736 nucleotides corresponding to nucleotide positions 18 to 754 of C. parvum with GenBank accession numbers KC748017-19 and 1 to 328 of $G$. intestinalis with GenBank accession numbers KC748020-23. Phylogenetic trees were constructed with Mega 5.10 software by using the maximum likelihood method and the Kimura two-parameter model with 1000 bootstrap sampling (Tamura et al. 2011). Cryptosporidium muris and Giardia muris sequences (JX948127 and AY258618, respectively) were used as the outgroups in order to root the trees.

\section{Results}

\section{Genotyping}

Sequence analysis of three Cryptosporidium 18S rDNA PCR products revealed the presence of three variants. One of the sequences (KC748019) was identical to the bovine genotype
BOH6 (AF093490) of C. parvum and other sequences originated from this species, isolated from: cattle (AB441687, EF611871, AY204237, AY204238, HQ009805, JX416362, JX298604, AB513870-81, AB513865-68, AF108864, JN120853), sheep (JN247404), deer (AF093494), alpaca (EF375894), rodents (HQ651731, GQ121019), and a fox (HQ822132), as well as in human patients (AJ849461, EU331237, EU331238, EU331241, GQ983351, GQ983355, JQ413434, HQ332160, AB434889, DQ067566, AJ493201, AB089290, AJ493544, AJ493547, DQ523504) from different countries. Two remaining sequences (KC748017 and $\mathrm{KC} 748018$ ) were unique and their similarity to AF093490 and the sequences mentioned above was $99.87 \%$.

According to the region of $\beta$-giardin gene PCR sequence analysis, three variants of $G$. intestinalis assemblage B were detected in all four positive samples. The similarity of these sequences to assemblage B sequence (HM165210) isolated from stool of patient and to other sequences obtained from humans from different countries (HM165210, DQ090522, DQ090523, DQ090525, DQ090527, DQ923579, AY072726, JQ684210, JQ684212, AY258616, AB618785, $\mathrm{AY} 072727)$ was $100 \%$ (KC748020 and KC748021) and $99 \%$ (KC748022 and KC748023).

\section{Phylogenetic analysis}

According to obtained phylogenetic tree (Fig. 1), the Cryptosporidium isolates analyzed in this study cluster together with the strain described as C. parvum bovine genotype and other $C$. parvum strains originated from cattle and humans. This clad forms a group to the sequences representing C. parvum ferret genotype and C. parvum mouse genotype I. The second large group contains the sequences originated from humans and a monkey described as $C$. parvum human genotype, the strain of $C$. hominis, as well as the sequence described as C. parvum rabbit genotype and the strain of Cryptosporidium cuniculus. The two large groups described above cluster together, as opposed to the third group containing a clad that consists of Cryptosporidium suis strain and two Cryptosporidium strains originated from pigs, and a clad containing Cryptosporidium ubiquitum strain, the strain described as $C$. parvum cervine genotype and the strain originated from a human.

The phylogenetic tree for Giardia (Fig. 2) shows that the sequences analyzed in this study cluster with sequences originated from human representing assemblage B of $G$. intestinalis. The clade consisting of these sequences clusters with the clade containing assemblage $\mathrm{E}$ sequences obtained from sheep and goat isolates. The rest of sequences used for tree construction comprise the big clade containing assemblage A sequences obtained from human and ruminants. 
Fig. 1 The phylogenetic tree for Cryptosporidium

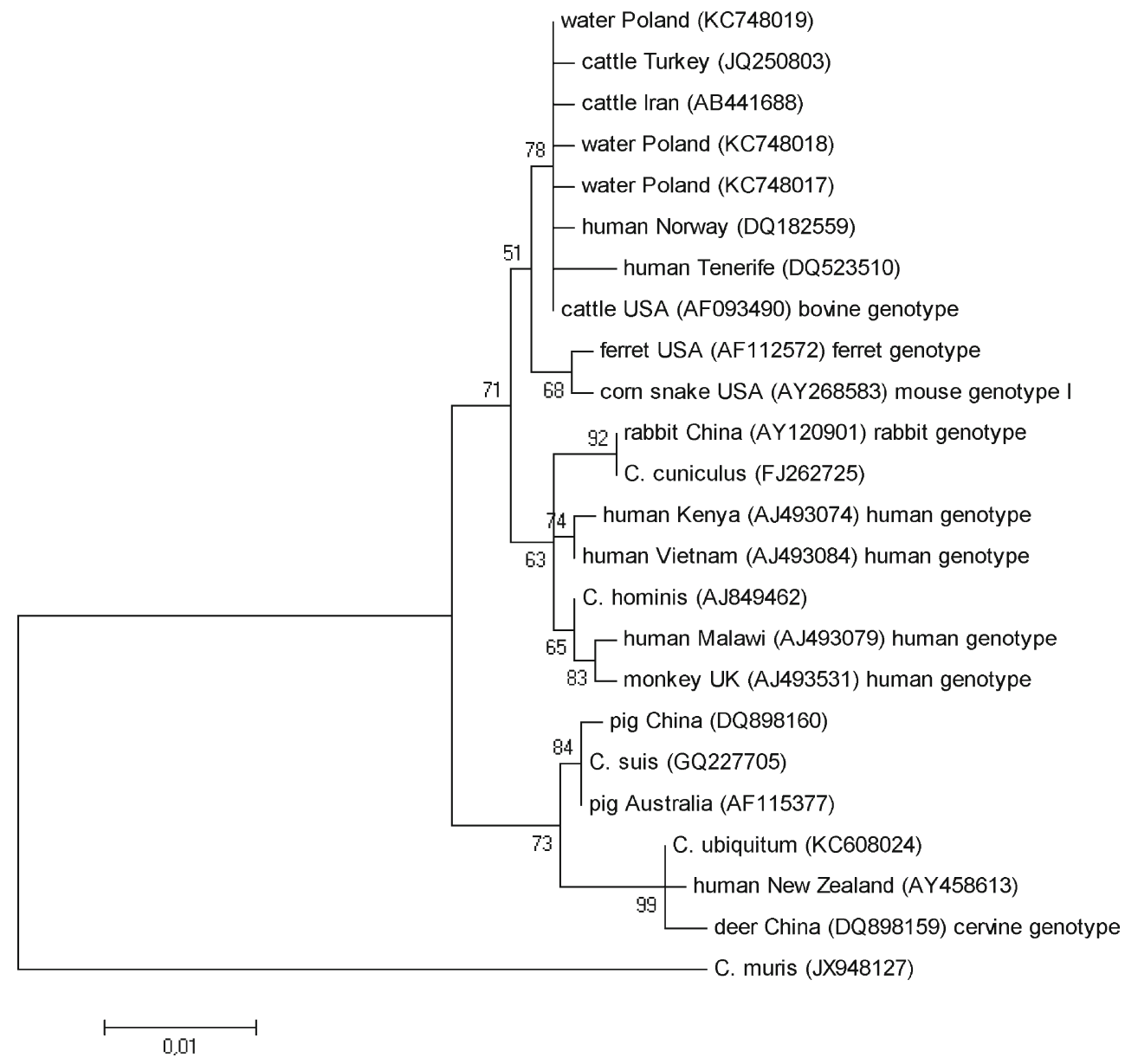

\section{Discussion}

Many water samples have been shown to contain Cryptosopridium oocysts, but not all the species and genotypes identified in water are health-threatening for human (Xiao and Feng 2008; Ruecker et al. 2012; Rossle and Latif 2013; Slapeta 2013). In this study, the DNA of Cryptosporidium oocysts from three lakes was analyzed, and in all the samples the presence of the bovine genotype of C. parvum was revealed. C. parvum is a multispecies complex containing several genotypes and some of them have been named as separate species, e.g., C. parvum human genotype was named $C$. hominis (Šlapeta 2006; Ren et al. 2012). The sequences that showed $100 \%$ or over $99 \%$ similarity with the sequences analyzed in this study were isolated from different mammals and from immunocompetent or HIV-infected human patients who had clinical symptoms of cryptosporidiosis (diarrhea) and/or the infection was confirmed by microscopy and direct immunofluorescence test (Gatei et al. 2002, 2003; Kváč et al. 2009; Muthusamy et al. 2006; Satoh et al. 2005; Šba et al. 2006). As the analysis of the sequences obtained from Polish lakes included relatively long fragment of $18 \mathrm{~S}$ rDNA, it is possible that the strains infective to human and animals were identified. All these sequences clustered together with the sequences representing C. parvum bovine genotype, isolated from cattle and human (Fig. 1), and this genotype grouped separately from the clad containing ferret and mouse genotypes of C. parvum, what is in accordance with results obtained by others (Šlapeta 2006, 2013; Ren et al. 2012) and supports a suggestion that the bovine genotype should be a separate species.

G. intestinalis is a complex species containing eight genotypes that are proposed to be distinct species due to the large genetic distances between them (Ryan and Cacciò 2013). These data are supported by the phylogenetic tree showed in this study (Fig. 2) that consists of two big clades - one containing assemblage B sequences isolated from humans and water, and the other, consisting of assemblage A sequences isolated from humans and other mammals. In the present study, four PCR products of Giardia $\beta$-giardin sequence were analyzed and the assemblage B of $G$. intestinalis was identified in all samples. In two samples, a sequence identical to 12 G. intestinalis sequences from GenBank obtained from humans was identified, whereas in remaining two samples unique sequences were detected and their similarity to the human-originating sequences was over $99 \%$. The sequences 


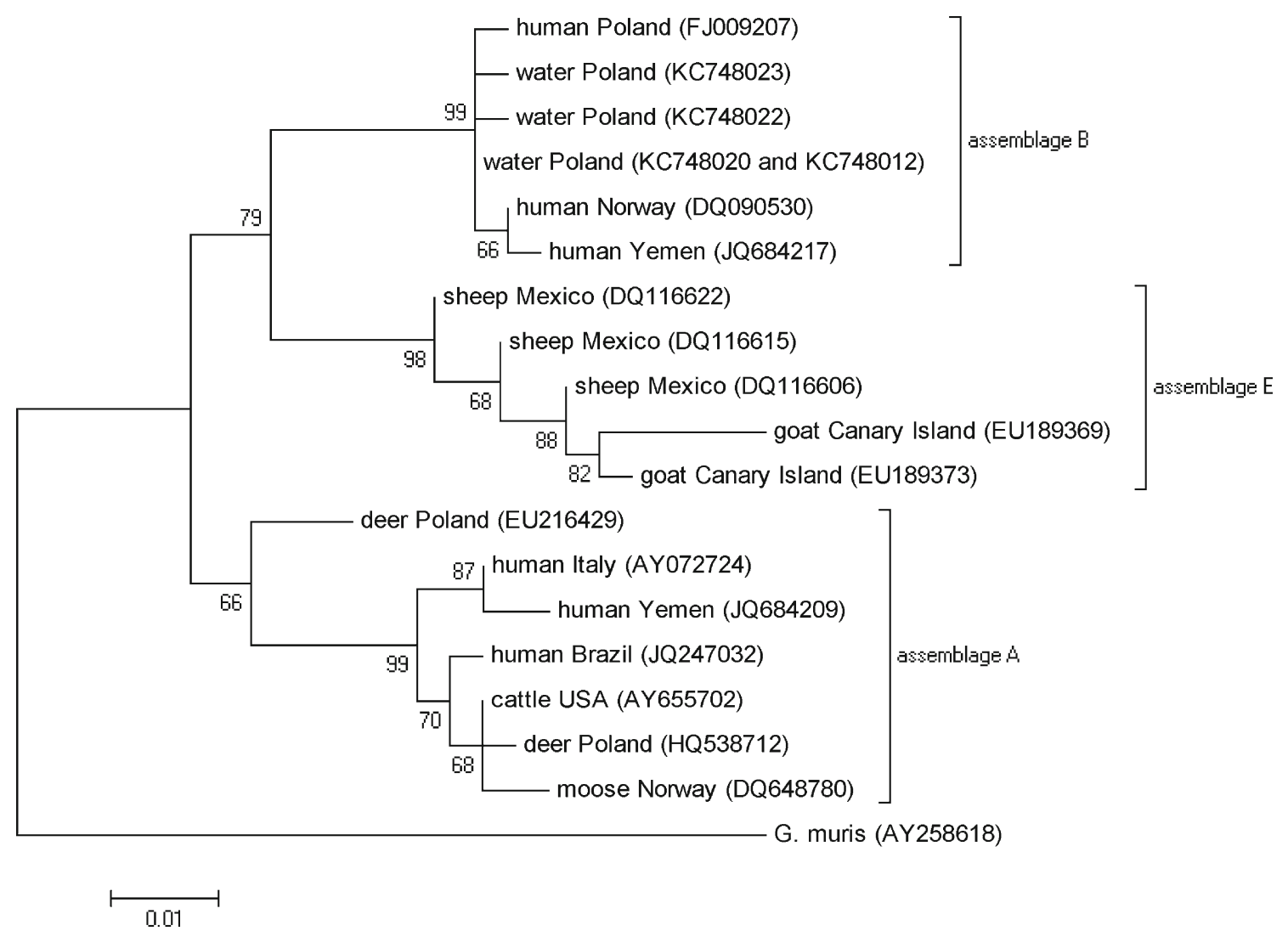

Fig. 2 The phylogenetic tree for Giardia

obtained by others from humans were isolated from stool of patients with diarrhea and giardiasis confirmed by different methods including microscopy, fecal antigen test, and immunofluorescence (Cacciò et al. 2002; Guy et al. 2004; Lebbad et al. 2011; Robertson et al. 2007; Abe and Teramoto 2012). It is not possible to determine a subassemblage of the sequences analyzed in this study, because they are much shorter than those obtained by others. On the other hand, it may be difficult even on the basis of multi-locus analysis because different subassemblages sequences of different loci were detected in the same isolates (Cacciò et al. 2002; Robertson et al. 2007).

Four of the five water bodies examined in this study are used for recreational activities (bathing, fishing, and sailing) during summer months, and the water samples were collected from the bathing places, so there is a risk of both cryptosporidiosis and giardiasis in north-western Poland. Although genotyping is crucial in determination of the (oo)cysts origin in water because of Cryptosporidium and Giardia host specificity, knowing the examined water bodies and their environment are also helpful. C. parvum (the bovine genotype) infects mainly human and pre-weaned calves, and dairy calves less than 2 months age are the major contributors of this zoonotic species. However, some epidemiological studies implicated farm (e.g., sheep) and companion (e.g., dogs and horses) animals as a source of human cryptosporidiosis (Hajdušek et al. 2004; Xiao and Fayer 2008; Xiao 2010; Chalmers et al. 2011). What is more, the source of $C$. parvum in human can be also of human origin and many cases of human C. parvum infections may be not zoonotic (Xiao and Fayer 2008; Xiao 2010). The host adaptation of Cryptosporidium spp. is not strict host specificity and cross-species transmissions may occur (Xiao and Feng 2008), e.g., natural infections with C. parvum bovine genotype have been also found in fox, red deer, and rodents (Hajdušek et al. 2004; Lv et al. 2009; Robinson et al. 2011). The host distribution of $G$. intestinalis assemblage $\mathrm{B}$ is associated mainly with human and other primates and to a much lesser extent with wildlife (e.g., foxes, wolves, beavers, or few birds species) and dogs (Dado et al. 2012; Ryan and Cacciò 2013). C. parvum bovine genotype and G. intestinalis assemblage B were detected in five lakes in north-western Poland. Dabie Duze and Dabie Male lakes are the parts of one large water body that adjoins mainly inhabited areas and they are used as sewage discharge places by the local house owners. Glebokie Lake is also a sewage discharge place and is located near a stud farm. Thus, the contamination of the three lakes originates most probably from human, especially in case of $G$. intestinalis assemblage B associated mainly with human. $C$. parvum bovine genotype is associated both with cattle and human; however, there are no cattle farms or fertilized cultivated fields near Glebokie and both Dabie Lakes. Horses may be a minor source of contamination in case of Glebokie Lake; nevertheless, they are hosts rather for the 
horse genotype of C. parvum than the bovine genotype (Xiao and Fayer 2008; Xiao 2010) and the assemblage B of G. intestinalis that may be present in horses (Ryan and Cacciò 2013) was not detected in this water body. Weltynskie Lake is surrounded by inhabited and recreational areas, as well as by Puszcza Bukowa forest and serves as a watering place for wild animals. In case of this water body, where assemblage B of $G$. intestinalis was identified, the contamination originates most likely from wildlife. Rusalka Lake is a little water body placed in a public garden near the centre of Szczecin; however, some species of wild animals (e.g., foxes or roe deer) are often observed in the park at night time, while during the day there are many household dogs in this area. Human and cattle are not a probable source of Cryptosporidium and Giardia (oo)cysts in this water body, thus, wild animals and domestic dogs seem to play this role.

\section{Conclusions}

Only molecular techniques allow detecting infectious Cryptosporidium and Giardia species and genotypes, so genetic characteristic of these protozoa occurring in water bodies is crucial to evaluate the health risk as well as to determine the contamination source due to the host specificity of these pathogens. However, non-specific relationship between hosts and pathogens may occur and the knowledge of the examined water bodies and their environment is also needed to define the source of contamination. In some cases, minor hosts may be of great importance in spreading Cryptosporidium and Giardia (oo)cysts in natural water reservoirs. In this study, such hosts seem to play the role of contamination sources of two from five examined lakes where pathogenic genotypes of C. parvum and G. intestinalis were found.

Acknowledgments This study was supported in part by the Ministry of Science and Higher Education, grant no. N N404 248635.

\section{Conflict of interest None declared}

Open Access This article is distributed under the terms of the Creative Commons Attribution License which permits any use, distribution, and reproduction in any medium, provided the original author(s) and the source are credited.

\section{References}

Abe N, Teramoto I (2012) Molecular evidence for person-to-person transmission of a novel subtype in Giardia duodenalis assemblage $\mathrm{B}$ at the rehabilitation institution for developmentally disabled people. Parasitol Res 110:1025-1028

Adamska M, Sawczuk M, Kolodziejczyk L, Skotarczak B (2014) Assessment of molecular methods as a tool for detecting of pathogenic protozoa isolated from water tanks, in press
Cacciò SM, De Giacomo M, Pozio E (2002) Sequence analysis of the $\beta$ giardin gene and development of a polymerase chain reactionRestriction fragment length polymorphism assay to genotype Giardia duodenalis cysts from human faecal samples. Int $\mathbf{J}$ Parasitol 32:1023-1030

Chalmers RM, Smith RP, Hadfield SJ, Elwin K, Giles M (2011) Zoonotic linkage and variation in Cryptosporidium parvum from patients in the United Kingdom. Parasitol Res 108:1321-1325

Dado D, Montoya A, Blanco MA, Miró G, Saugar JM, Bailo B, Fuentes I (2012) Prevalence and genotypes of Giardia duodenalis from dogs in Spain: Possible zoonotic transmission and public health importance. Parasitol Res 111:2419-2422

Gatei W, Suputtamongkol Y, Waywa D, Ashford RW, Bailey JW, Greensill J, Beeching NJ, Hart CA (2002) Zoonotic species of Cryptosporidium are as prevalent as the anthroponotic in HIV-infected patients in Thailand. Ann Trop Med Parasitol 96(8):797-802

Gatei W, Greensill J, Ashford RW, Cuevas LE, Parry CM, Cunliffe NA, Beeching NJ, Hart CA (2003) Molecular analysis of the 18S rRNA gene of Cryptosporidium parasites from patients with or without human immunodeficiency virus infections living in Kenya, Malawi, Brazil, the United Kingdom, and Vietnam. J Clin Microbiol 41(4): 1458-1462

Guy RA, Xiao C, Horgen PA (2004) Real-Time PCR assay for detection and genotype differentiation of Giardia lamblia in stool specimens. J Clin Microbiol 42(7):3317-3320

Hajdušek O, Ditrich O, Šlapeta J (2004) Molecular identification of Cryptosporidium spp. in animal and human hosts from the Czech Republic. Vet Parasitol 122:183-192

Kváč M, Květoňová D, Sak B, Ditrich O (2009) Cryptosporidium pig genotype II in immunocompetent man. Emerg Infect Dis 15(6):982983

Lebbad M, Petersson I, Karlsson L, Botero-Kleiven S, Andersson JO, Svenungsson B, Svärd SG (2011) Multilocus genotyping of human Giardia isolates suggests limited zoonotic transmission and association between assemblage B and flatulence in children. PLoS Negl Trop Dis 5(8): 1262

Lv C, Zhang L, Wang R, Jian F, Zhang S, Ning C, Wang H, Feng C, Wang X, Ren X, Qi M, Xiao L (2009) Cryptosporidium spp. in wild, laboratory, and pet rodents in China: Prevalence and molecular characterization. Appl Environ Microbiol 75(24):7692-7699

Muthusamy D, Rao SS, Ramani S, Monica B, Banerjee I, Abraham OC, Mathai DC, Primrose B, Muliyil J, Wanke CA, Ward HD, Kang G (2006) Multilocus genotyping of Cryptosporidium sp. isolates from human immunodeficiency virus-infected individuals in South India. J Clin Microbiol 44(2):632-634

Ren X, Zhao J, Zhang L, Ning C, Jian F, Wang R, Lv C, Wang Q, Arrowood MJ, Xiao L (2012) Cryptosporidium tyzzeri n. sp. (Apicomplexa: Cryptosporidiiae) in domestic mice (Mus musculus). Exp Parasitol 130:274-281

Robertson LJ, Forberg T, Hermansen L, Gjerde BK, Langeland N (2007) Molecular characterisation of Giardia isolates from clinical infections following a waterborne outbreak. J Infect 55:79-88

Robinson G, Chalmers RM, Stapleton C, Palmer SR, Watkins J, Francis C, Kay D (2011) A whole water catchment approach to investigating the origin and distribution of Cryptosporidium species. J Appl Microbiol 111(3):717-730

Rossle NF, Latif B (2013) Cryptosporidiosis as threatening health problem: a review. Asian Pac J Trop Biomed 3(11):916-924

Ruecker NJ, Matsune JC, Wilkes G, Lapen DR, Topp E, Edge TA, Sensen CW, Xiao L, Neumann NF (2012) Molecular and phylogenetic approaches for assessing sources of Cryptosporidium contamination in water. Water Res 46(16):5135-5150

Ryan U, Cacciò SM (2013) Zoonotic potential of Giardia. Int J Parasitol 43:943-956

Satoh M, Kimata I, Iseki M, Nakai Y (2005) Gene analysis of Cryptosporidium parvum HNJ-1 strain isolated in Japan. Parasitol Res 97:452-457 
Šlapeta J (2006) Cryptosporidium species found in cattle: a proposal for new species. Trends Parasitol 22(10):496-474

Šlapeta J (2013) Cryptosporidiosis and Cryptosporidium species in animals and humans: a thirty colour rainbow? Int J Parasitol 43:957-970

Šoba B, Petrovec M, Mioč V, Logar J (2006) Molecular characterization of Cryptosporidium isolates from humans in Slovenia. Clin Microbiol Infect 12(9):918-921

Tamura K, Peterson D, Peterson N, Stecher G, Nei M, Kumar S (2011) MEGA5: molecular evolutionary genetics analysis using maximum likelihood, evolutionary distance, and maximum parsimony methods. Mol Biol Evol 28(10):2731-2739

Xiao L (2010) Molecular epidemiology of cryptosporidiosis: an update. Exp Parasitol 124:80-89

Xiao L, Fayer R (2008) Molecular characterization of species and genotypes of Cryptosporidium and Giardia and assessment of zoonotic transmission. Int J Parasitol 38:1239-1255

Xiao L, Feng Y (2008) Zoonotic cryptosporidiosis. FEMS Immunol Med Microbiol 52:309-323 